\title{
Biotipo Morfológico Facial en Tres Grupos Étnicos Colombianos: Una Nueva Clasificación por Medio del Índice Facial
}

\author{
Facial Biotype in Three Colombian Ethnic Groups: a New Classification by Facial Index \\ Bedoya, A.; Osorio, J. C. \& Tamayo, J. A.
}

BEDOYA, A.; OSORIO, J. C. \& TAMAYO, J. A. Biotipo morfológico facial en tres grupos étnicos colombianos: una nueva clasificación por medio del índice facial. Int. J. Morphol., 30(2):677-682, 2012.

RESUMEN: El objetivo de este estudio es evaluar el índice facial de tres poblaciones colombianas y proponer un nuevo método para identificar los biotipos morfológicos faciales teniendo en cuenta el tipo de ascendencia. Para esto, se tomaron las medidas de Nasion a Gnation y la distancia Bicigomática con un calibrador digital en tres grupos étnicos diferentes: 63 niños de la población Ticuna (Amazonas), 68 niños del municipio de Puerto Tejada (Cauca) y 65 niños del municipio de Santiago de Cali (Valle). Por medio de la prueba post hoc T2 de Tamhane se determinó que las poblaciones son distintas y que el índice morfológico facial tradicional no discriminaba esas diferencias. Por tal razón se utilizó el método estadístico de conglomerados difusos con el fin de determinar el número de grupos o biotipos para cada tipo de ascendencia. Se concluyó, que las poblaciones mestizas y afro-descendientes tienen similitudes por lo cual se agruparon juntas y el algoritmo c-medias generó cuatro biotipos característicos, mientras que para la población indígena Ticuna (Amazonas) solo se generaron tres biotipos.

PALABRAS CLAVE: Biotipo; Ascendencia; Índice facial; Conglomerados difusos.

\section{INTRODUCCIÓN}

El control del crecimiento craneofacial requiere procesos biológicos precisos que regulan la iniciación y dirección de los mecanismos, patrones y velocidades de crecimiento (Moss, 1997) La regularidad con la cual la cara de un niño crece, y mantiene una morfología y semejanza a sus familiares, sugiere que los factores genéticos tienen una fuerte influencia en el crecimiento craneofacial (Kohn, 1991). La morfología craneofacial es considerada ahora multifactorial, es decir, el desarrollo facial es influenciado por un número de genes y por varios factores medioambientales (Johannsdottir et al., 2005).

Este tipo de herencia multifactorial, que regula el crecimiento craneofacial, se ve fuertemente influenciada por aspectos culturales y geográficos de las poblaciones (Feldman \& Laland, 1996). Estas diferentes tasas de crecimiento y desarrollo craneofacial entre diferentes grupos poblacionales permite generar patrones que forman y delimitan los diferentes biotipos faciales (Feldman \& Laland).

Varios autores han confirmado la importancia del biotipo facial para la planificación del tratamiento y para el pronóstico del mismo (Björk, 1947; Broadbent, 1937;
Brodie, 1941; Chang et al., 1997; Downs, 1956; Ricketts, 1960), ya que si el paciente tuviese un remanente de crecimiento, el biotipo y por ende las distintas formas en que se modifican los componentes craneofaciales, pueden alterar la estabilidad del tratamiento en el tiempo. Además, se ha proporcionado evidencia sobre el efecto que tiene la etnia sobre las variaciones faciales en los distintos biotipos (Lee et al., 1997; Lu et al., 2005; Ramanathan et al., 2009).

La identificación del biotipo facial se ha estudiado desde comienzos del siglo XIX, haciendo referencia en esa época, a las diferencias raciales que enfatizaron la superioridad de las poblaciones humanas europeas (Cameron, 1929). En 1957 Martin \& Saller determinan el biotipo facial como la anchura desde el cigomático derecho al cigomático izquierdo multiplicada por cien y dividida por la altura de la cara desde el punto nasión hasta el punto mentón (Rakosi \& Irmtrud, 1992). Muchos estudios han utilizado este índice para determinar el biotipo facial pero no tienen en cuenta los diferentes tipos de ascendencia.

En este estudio se propone la utilización de la técnica estadística de conglomerado difuso y su aplicación en la 
clasificación del biotipo morfológico facial, tomando el Índice Morfológico Facial (IMF) propuesto por Martin y Saller pero adicionando, según la ascendencia, el grado de pertenencia de los individuos estudiados a los grupos que se forman a partir de los conglomerados difusos. Por esta razón el objetivo de este estudio es proponer un nuevo método para identificar los biotipos morfológicos faciales teniendo en cuenta el tipo de ascendencia y utilizarlo en tres poblaciones colombianas.

\section{MATERIAL Y MÉTODO}

Participantes.La muestra fue intencional y estuvo conformada por 63 niños de la comunidad indígena Ticuna, ubicada en Leticia (Amazonas), 68 niños afrodescendientes del municipio Puerto Tejada (Cauca) y 65 niños de la población mestiza del municipio de Santiago de Cali (Valle del Cauca), conformando un total de 196 niños estudiados. Todos los niños evaluados tenían edades entre 12 y 14 años. Se obtuvo el consentimiento informado por parte de los padres de familia $o$ acudientes de los menores, al igual que de las directivas del colegio y el asentimiento indígena por parte de los niños y niñas con capacidad de discernimiento y de aceptación. Esta investigación fue aprobada por el Comité de Ética de la Institución Universitaria Colegios de Colombia UNICOC y según Resolución 8430 del Ministerio de Salud de la República de Colombia. Además no se consideró la existencia de algún tipo de riesgo al participar en el proyecto.

Criterios de inclusión y exclusión. Como criterios de inclusión se tuvo en cuenta que se pudieran demostrar tres generaciones de ancestría tanto para la población afrodescendiente como amerindia. Se excluyeron de este estudio aquellos individuos que presentaran malformaciones congénitas y que estuvieran por fuera del rango etario.

Índice Morfológico Facial. Se obtuvieron dos medidas clínicas de la cara a cada uno de los niños seleccionados: 1) del Nasion (N) a Gnation (Gn) y 2) de Cigomático derecho (Cgd) a Cigomático izquierdo (Cgi).

Posteriormente se calculó el índice morfológico facial (IMF). Este se obtiene a partir de la relación entre la altura morfológica de la cara (de $\mathrm{N}$ a Gn) y la anchura bicigomática (de Cgd a Cgi) (Rakosi \& Irmtrud):

Clasificación del biotipo facial por medio de los conglomerados difusos. El término difuso procede de la expresión Fuzzy que viene del inglés Fuzz (tamo, pelusa, vello) y se traduce por difuso o borroso. Los conjuntos difusos fueron introducidos por Zadeh (1965) para procesar, manipular información y datos afectados de incertidumbre e imprecisión no probabilística. La idea de Zadeh es hacer que el rango de valores de pertenencia de un elemento a un conjunto pueda variar en el intervalo $[0,1]$ en lugar de limitarse a uno de los valores del par (Dunn, 1973) (o lo que es lo mismo Falso, Verdadero).

El análisis de conglomerados difuso tiene sus orígenes en 1973 (Bezdek, 1981), y fue posteriormente generalizado en Bezdek (1981), que introdujo el método de fuzzy c means (Kohn). Para el caso de estudio, este método nos permitió establecer formalmente la mejor partición o puntos de corte del rango del índice morfológico facial tomado a partir de la medida vertical N-ng y la medida horizontal desde cigomático derecho a cigomático izquierdo y así mismo asignar a cada individuo, según su ascendencia, un grado de pertenencia a cada uno de los grupos formados.

Análisis estadístico. Los resultados fueron analizados con el programa estadístico R: A language and environment for statistical computing. Para identificar si existen diferencias en el IMF en función de las tres ascendencias estudiadas, se utilizó la prueba no paramétrica de Kruskal-Wallis, dado que el contraste de normalidad Shapiro Wilks para la variable IMF arrojó un valor que no mostraba evidencia sobre la normalidad (p-valor $<0,05$ ). Se estableció un nivel de significación del 5\%.

Se hizo uso de tres análisis de conglomerados difusos, cada uno corresponde al IMF de cada ascendencia. Por tanto se clasificó en grupos a los sujetos estudiados dependiendo de su índice fácil. La clasificación se realizó por medio del algoritmo C-Medias Difuso, a través de la librería e1071 del programa estadístico $\mathrm{R}$.

\section{RESULTADOS}

En el estudio participaron 196 niños, de los cuales, según la clasificación propuesta por Martin y Saller, todos los de ascendencia Mestiza son de cara alargada. Los niños de ascendencia afro-descendiente presentan en su mayoría un biotipo de cara alargada $(98,5 \%)$ mientras que solo una muy pequeña proporción presentan una cara corta $(1,5 \%)$. Para la población Indígena Ticuna (Amazonas) el 76,2\% presentan una cara alargada y un $23,8 \%$ presentan una cara corta (Tabla I).

$\mathrm{Al}$ comprobar si existen diferencias entre el IMF según los tres grupos de ascendencia a través de la prueba no paramétrica de Kruskal-Wallis, al nivel de significación del $5 \%$, se encontraron diferencias estadísticamente significativas ( $\mathrm{p}$-valor $<0,05$ ), es decir, que la morfología facial en los 
Tabla I. Índice morfológico facial según Martin y Saller para determinar el biotipo facial en tres ascendencias.

\begin{tabular}{llcc}
\hline Ascendencia & & Frecuencia & Porcentaje \\
\hline Mestizo & Cara Alargada & 65 & 100,0 \\
Afro & Cara Corta & 1 & 1,5 \\
& Cara Alargada & 67 & 98,5 \\
& Total & 68 & 100 \\
Indígenas & Cara Corta & 15 & 23,8 \\
& Cara Alargada & 48 & 76,2 \\
& Total & 63 & 100 \\
\hline
\end{tabular}

tres grupos de ascendencia no es el mismo. La prueba post hoc T2 de Tamhane evidencia que los grupos de ascendencia mestizo y afrodescendiente tienen una morfología facial muy similar (p-valor=0,515) y a su vez una morfología facial mucho más alargada que el grupo de niños con ascendencia indígena ( $\mathrm{p}$-valor=0,00). Esto muestra, que la clasificación propuesta por Martin \& Saller (Rakosi \& Irmtrud), no es aplicable para todos los grupos de ascendencia, puesto que cada una de ellas tiene una tipología de morfología facial diferente (Tabla II). Por esta razón, en este estudio se agrupo los mestizos con los afro-descendientes para determinar en cuantos biotipos se divide este conjunto de individuos.

Clasificación propuesta para el biotipo facial.

Tabla II. Prueba Kruskal Wallis que evalúa las diferencias en el IMF según la ascendencia.

\begin{tabular}{lccc}
\hline & Media & Desviación típica & p-valor \\
Mestizo & 103,4 & 8,6 & \\
Afrodescendiente & 105,3 & 8,6 & 0,000 \\
Indígenas & 85,4 & 2,3 & \\
\hline
\end{tabular}

Grupo de ascendencia mestiza y afrodescendiente. Se realizaron varias corridas del algoritmo C-Medias Difuso considerando varios tamaños de clases o categorías de biotipos ( $c=2,3$ y 4), con el fin de encontrar por medio del Índice de Partición Difusa (FPI) el número adecuado de clases, que para este caso representará el número de categorías del IMF. La Tabla III muestra que c=4 es el número de clases optimo a escoger, ya que obtiene el valor FPI menor, es decir, que los datos evidencian que el número de biotipos faciales más apropiado para estos grupos de ascendencia son cuatro. Esto quiere decir, que este grupo no comparte un mismo biotipo sino que se requiere agruparlos en cuatro biotipos.

Tabla III. FPI para cada clase o categorías de biotipos.

\begin{tabular}{lcc}
\hline Número de clases & F & FPI \\
\hline 2 & 0,8371609 & 0,3615759 \\
3 & 0,7756037 & 0,3365945 \\
4 & 0,7288181 & 0,3256783 \\
\hline
\end{tabular}

Cada una de las clases está centrada sobre el valor obtenido por el procedimiento, permitiendo asignar una etiqueta que sirva para reconocer un grupo de sujetos de ascendencia mestiza o afrodescendiente con morfología facial muy similar. Los resultados de los centros de clases y la distribución de sujetos en cada clase se muestran en la Tabla IV. Según los centros de clases podemos denominar a la primera clase como el conjunto de individuos con un IMF más pequeño, y la cuarta clase como el conjunto de individuos con un IMF más alto. Se observa que el 11,3\% de la muestra estudiada tiene una cara más corta, mientras que tan solo el $23,3 \%$ tienen una cara más alargada con respecto a los sujetos estudiados. La clasificación propuesta para el biotipo facial en estos grupos de ascendencia (mestizo y afrodescendientes) con sus respectivos puntos de corte, es la que se muestra en la Tabla V.

Tabla IV. Centros de clases y distribución de sujetos de grupo ascendente mestizo y afrodescendiente en las categorías de biotipos conformados.

\begin{tabular}{lccc}
\hline Clase & Centros & Frecuencia & Porcentaje \\
Clase 1 & 91,09065 & 15 & 11,27 \\
Clase 2 & 98,84660 & 44 & 33,08 \\
Clase 3 & 106,69581 & 43 & 32,33 \\
Clase 4 & 114,57854 & 31 & 23,30 \\
\hline
\end{tabular}

Tabla V. Clasificación propuesta para el biotipo facial en mestizos y afrodescendientes.

\begin{tabular}{lc}
\hline Clase & Puntos de corte \\
Clase 1 & $\mathrm{IMF} \leq 95$ \\
Clase 2 & $95,1 \leq \mathrm{IMF} \leq 102,9$ \\
Clase 3 & $103 \leq \mathrm{IMF} \leq 110,9$ \\
Clase 4 & $111 \mathrm{IMF} \geq$ \\
\hline
\end{tabular}

Grupo de ascendencia indígena. La tabla VI muestra que c=3 es el número de categorías óptimo para la estructura del biotipo facial de este grupo de ascendencia ya que se obtiene el valor FPI más pequeño. Este resultado difiere con respecto a los mestizos y afro-descendientes.

\begin{tabular}{lcc}
\multicolumn{3}{l}{ Tabla VI. FPI para cada clase o categorías de biotipos. } \\
\hline Número de clases & F & FPI \\
\hline $\mathbf{2}$ & 0,8184936 & 0,3630128 \\
$\mathbf{3}$ & 0,8452155 & 0,2321767 \\
$\mathbf{4}$ & 0,7858297 & 0,2855605 \\
\hline
\end{tabular}


En la Tabla VII se resume los centros de clase y la distribución de sujetos en las dos clases conformadas. Se muestra que el $36,5 \%$ de los niños indígenas estudiados tienen un IMF bajo, mientras que el resto tienen un biotipo facial de una cara mucho más larga. Se destaca que un niño tiene un IMF mucho más alto que el resto de su misma ascendencia. Finalmente, la clasificación propuesta para el biotipo facial en este grupo de ascendencia indígena con sus respectivos puntos de corte, se muestra en la Tabla VIII.

Tabla VII. Centros de clases y distribución de sujetos de grupo ascendente indígena en las categorías de biotipos conformados.

\begin{tabular}{lccc}
\hline Clase & Centros & Frecuencia & Porcentaje \\
\hline Clase 1 & 83,90106 & 23 & 36,50 \\
Clase 2 & 86,14001 & 39 & 61,90 \\
Clase 3 & 99,98973 & 1 & 1,60 \\
\hline
\end{tabular}

Tabla VIII. Clasificación propuesta para el biotipo facial en niños indígenas.

\begin{tabular}{lc}
\hline Clase & Puntos de corte \\
\hline Clase 1 & $\mathrm{IMF} \leq 84,9$ \\
Clase 2 & $85 \leq \mathrm{IMF} \leq 93,9$ \\
Clase 3 & $94 \leq \mathrm{IMF}$ \\
\hline
\end{tabular}

\section{DISCUSIÓN}

Al utilizar el índice morfológico facial según Martin \& Saller sin tener en cuenta el tipo de ascendencia la mayoría de los individuos de una población presentaron un solo biotipo facial. Por ejemplo para la población mestiza el 100 por ciento de los individuos analizados presentaron una cara alargada. Las características faciales y el desarrollo facial de los individuos siguen complejos patrones de desarrollo y están modulados por una gran cantidad de genes que a su vez están fuertemente controlados por el ambiente (PiquetThépot, 1962). Esto quiere decir que es poco probable que una característica como el biotipo tenga un solo fenotipo en la población. Los rasgos cuantitativos y multifactoriales por lo regular muestran una distribución diferente para la característica evaluada. Por lo que un método para establecer el biotipo facial debería poder clasificar esas diferencias.

Cuando utilizamos la clasificación del biotipo facial por medio de los conglomerados difusos nos permitió agrupar según la ascendencia los individuos que tuvieran un grado de pertenencia a unos de los subgrupos formados. Lo primero que se encontró fue que no se evidencian suficientes diferencias para separar la población mestiza de Santiago de Cali con la población afro-descendiente de Puerto Tejada. Geográficamente estas dos poblaciones no están tan separadas y a través del tiempo han tenido un continuo flujo génico lo que hace posible que compartan algunas características que generen un biotipo facial compartido. El biotipo facial para estas dos poblaciones, de acuerdo con este trabajo, quedó dividido en cuatro subgrupos o clases, y se propone que los individuos que tengan un IMF $\leq 95$ se clasifiquen como cara muy corta, los individuos con una rango $95,1 \leq \mathrm{IMF} \leq$ 102,9 tendrían una cara corta, los individuos con un rango $103 \leq \mathrm{IMF} \leq 110,9$ serían cara intermedia y los individuos con IMF $\geq 111$ tendrían un cara alargada. De esta forma el $33 \%$ de los individuos pertenecientes a estas dos poblaciones tendrían caras cortas y un 32,3\% intermedias. Al comparar estas poblaciones con la población de la isla de Córcega (Mediterráneo) en donde se determinó que la distribución de los individuos era de 25 porciento euriprosopos, 25 porciento mesoprosopos, 30 porciento leptoprosopos y 20 porciento hiperleptoprosopos (Beecher \& Corruccini, 1981), se puede ver algún grado de similitud con las poblaciones mestiza y afro-descendiente evaluada, ya que esta isla tiene un alto predominio latino y una fuerte relación con las costas africanas del Mediterráneo.

La población Ticuna del Amazonas presentó un comportamiento diferente al de las poblaciones Mestizas y Afrodescendientes. Desde el punto de vista del flujo génico, este se va a ver muy limitado tanto geográficamenate como culturalmente. Además el efecto que tiene la alimentación en el desarrollo de las estructuras faciales va a generar mayores diferencias (del Sol, 2006). Para esta población se encontraron tres clases o categorías con parámetros diferentes a las dos poblaciones antes evaluadas. Para esta población, los individuos con un IMF $\leq 84,9$ se clasificarían como cara corta, aquellos con $85 \leq \mathrm{IMF} \leq 93,9$ tendrían una cara intermedia y los que tengan un IMF $>94$ serían caras largas. Con esta clasificación casi el 62\% de los individuos de esta población serían cara intermedia y casi un 36,5\% tendrían una cara corta. Se comparó este estudio con uno hecho para índice facial en el grupo étnico mapuche, en donde la población en general presentó individuos con un rostro mediano a ancho y bajo (Ramos Pazos et al., 2007). Después de utilizar nuestro índice, la población indígena Ticuna siguió manteniendo en general un rostro mediano, y mantiene cierto parecido con la población mapuche.

Las relaciones faciales transversales y verticales presentan variaciones entre las distintas etnias, siendo este conocimiento relevante en el campo de las cirugías plástica y maxilofacial, la antropología y la medicina forense (Forster et al., 2008). De igual forma la relación existente entre el arco dental y el modelo facial vertical es importante consi- 
derarla, ya que pacientes con cara larga tienden a tener arcos dentales angostos y pacientes de cara corta tienden a tener arcos dentales anchos. En general, para la planificación de los tratamientos ortodónticos en nuestra población se hace necesario la determinación del biotipo facial individual, ya que este está directamente relacionado con el tamaño de los arcos, entre otras características y el estudio de nuestras poblaciones permitirá tener una práctica clínica contextualizada a nuestra realidad.

En conclusión, para la población mestiza del Valle del Cauca y afro-desdiente de Puerto Tejada (Cauca), las cuales son muy similares, se recomienda cuatro biotipos fáciles: cara muy corta, cara corta, cara intermedia y cara larga. La población indígena amazónica tiene un biotipo facial muy distinto a las poblaciones del sur occidente co- lombiano evaluadas y recomendamos utilizar tres biotipos: cara corta, cara intermedia y cara larga.

Se identificó el efecto que tiene la ascendencia sobre el biotipo facial de los individuos y además se logra justificar la falta de exactitud que tiene IMF cuando no se tiene en cuenta los tipos de ascendencia.

Cada población tiene fuerzas micro-evolutivas diferentes, por lo cual las frecuencias de determinadas características varían considerablemente.

Las poblaciones son dinámicas, por lo cual es conveniente estudiarlas y constantemente ajustar los parámetros que consideramos a la hora de realizar tratamientos médi$\cos$.

BEDOYA, A.; OSORIO, J. C. \& TAMAYO, J. A. Facial biotype in three colombian ethnic groups: a new classification by facial index. Int. J. Morphol., 30(2):677-682, 2012.

SUMMARY: The aim of this study is evaluate the facial index of three Colombian populations and propose a new method to identify the facial morphological biotypes taking into account the type of ascendancy. For this, measures were taken of Nasion to Gnation and bizygomatic distance with a digital caliper in three ethnic groups: 63 children from Ticuna population (Amazonas), 68 children from municipality of Puerto Tejada (Cauca) and 65 children from municipality Santiago de Cali (Valle). Through the post hoc Tamhane's T2 was determined that the populations are different and the facial morphological index did not discriminate these differences. We used the statistical method of fuzzy clusters to determine the number of groups or biotypes for each type of ascendancy. We concluded that mixed populations and African descent have similarities, therefore they were grouped together and the c-means algorithm generated four biotypes characteristic, while the indigenous population Ticuna (Amazon) only generated three biotypes.

KEY WORDS: Biotype; Ascendancy; Facial index; Fuzzy cluster.

\section{REFERENCIAS BIBLIOGRÁFICAS}

Cameron, J. A study of the upper facial index in diverse racial types of mankind. Craniometric studies, no. 23. Am. J. Phys. Anthropol., 13:344-52, 1929.

Beecher, R. M. \& Corruccini, R. S. Effects of Dietary Consistency on Craniofacial and Occlusal Development in the Rat. Angle Orthod., 51(1):61-9, 1981.

Bezdek, J. Pattern Recognition with Fuzzy Objective Function Algorithms. New York, Plenum Press, 1981.

Björk, A. The face in profile. An Anthropological X-ray Investigation on Swedish Children and Conscripts. Svensk Tandläkare-Tidskrift, 40(5B):180, 1947

Broadbent, B. The face of the normal child. Angle Orthod., 7:183208, 1937.

Brodie, A. G. On the growth pattern of the human head. From the third month to the eighth year of life. Am. J. Anat., 68:209-62, 1941.
Chang, J. Y.; McNamara Jr, J. A. \& Herberger, T. A. A longitudinal study of skeletal side effects induced by rapid maxillary expansion. Am. J. Orthod. Dentofacial Orthop., 112(3):330-7, 1997.

del Sol, M. Índices Faciales en Individuos Mapuche. Int. J. Morphol., 24:587-90, 2006.

Downs, W. Analysis of the dentofacial profile. Angle Orthod., 26:191-212, 1956.

Dunn, J. A Fuzzy Relative of the ISODATA Process and Its Use in Detecting Compact WellSeparated Clusters. J. Cybernet., 3:3257, 1973.

Feldman, M. W. \& Laland, K. N. Gene-culture coevolutionary theory. Trends Ecol. Evol., 11:453-7, 1996.

Forster, C. M.; Sunga, E. \& Chung, C. H. Relationship between dental arch width and vertical facial morphology in untreated adults. Eur. J. Orthod., 30:288-94, 2008. 
BEDOYA, A.; OSORIO, J. C. \& TAMAYO, J. A. Biotipo morfológico facial en tres grupos étnicos colombianos: una nueva clasificación por medio del índice facial Int. J. Morphol., 30(2):677-682, 2012.

Johannsdottir, B.; Thorarinsson, F.; Thordarson, A. \& Magnusson, T. E. Heritability of craniofacial characteristics between parents and offspring estimated from lateral cephalograms. Am. J. Orthod. Dentofacial Orthop., 127(2):260-1, 2005.

Kohn, L. The Role of Genetics in Craniofacial Morphology and Growth. Annual Rev. Anthropol., 20:261-78, 1991.

Lee, J. J.; Ramirez, S. G. \& Will, M. J. Gender and racial variations in cephalometric analysis. Otolaryngol. Head Neck Surg., 117:326-9, 1997.

Lu, X.; Chen, H. \& Jain, A. Multimodal Facial Gender and Ethnicity Identification. In: Advances in Biometrics. Zhang, D. \& Jain, A. (Eds.). Vol. 3832. Berlin, Springer, 2005. pp.554-61.

Moss, M. L. The functional matrix hypothesis revisited. 4. The epigenetic antithesis and the resolving synthesis. Am. J. Orthod. Dentofacial Orthop., 112(4):410-7, 1997.

Piquet-Thépot, M. M. Étude anthropologique de la population corse ; note sur 22 sujets de la région de Calvi. Bull. Mem. Soc. Anthropol. Paris, 229-34, 1962.

Rakosi, T. \& Irmtrud, J. Atlas de Ortopedia Maxilar: Diagnóstico. Barcelona, Salvat, 1992.

Ramanathan, N.; Chellappa, R. \& Biswas, S. Computational methods for modeling facial aging: A survey. J. Vis. Lang. Comput., 20:131-44, 2009.

Ramos Pazos, N. A.; Suazo Galdames, I. C.; Martínez, M. L. M. \& Reyes, L. Relaciones Transversales Faciales en Niños Chilenos de la Región del Maule. Int. J. Morphol., 25:703-7, 2007.

Ricketts, R. M. Cephalometric synthesis: An exercise in stating objectives and planning treatment with tracings of the head roentgenogram. Am. J. Orthod., 46:647-73, 1960.

Zadeh, L. A. Fuzzy sets. Inf. Control, 8:338-53, 1965.

\section{Dirección para correspondencia: \\ Dr. Antonio Bedoya \\ Postgrado de Ortodoncia y Ortopedia Maxilar \\ Institución Universitaria Colegios de Colombia (UNICOC) \\ Universidad del Valle \\ COLOMBIA}

Email: determinadaarea@yahoo.com

Recibido : 15-06-2011

Aceptado: 28-02-2012 